\title{
"Do Bugs Need Drugs?" A community education program for the wise use of antibiotics
}

\author{
Carson $\mathrm{M}^{1^{*}}$, Patrick $\mathrm{DM}^{2,3}$
}

\author{
${ }^{1}$ Alberta Health Services, Edmonton, Alberta \\ ${ }^{2}$ UBC School of Population and Public Health, Vancouver, BC \\ ${ }^{3}$ British Columbia Centre for Disease Control, Vancouver BC
}

*Correspondence: mary.carson@albertahealthservices.ca

\begin{abstract}
"Do Bugs Need Drugs?" is a community and professional education program designed to address antibiotic resistance by decreasing the inappropriate use of antibiotics. Resources have been developed for physicians, pharmacists, nurses and the public, including children, their parents and caregivers, teachers, employers and workers, and long-term care facilities. There are four key strategies: consistent messaging (e.g., handwashing is the best way to stop the spread of infections); networking (through dedicated committee members who have engaged government ministries, professional organizations, health care organizations, academia, industry and businesses, and community groups); aligning interests (e.g., engaging nursing and medical students to deliver a program on antimicrobial resistance (AMR) to Grade 2 students as part of their community health curriculum); and containing costs (e.g., partnering with other organizations for distribution of materials and sharing administrative and printing costs between the Do Bugs Need Drugs programs in Alberta and British Columbia). There is evidence now of an increased willingness in the scientific and medical community to discuss the risks associated with antibiotic use and of growing public awareness that AMR is linked to misuse and overuse of antibiotics.
\end{abstract}

\section{Introduction}

Antibiotic-resistant organisms are responsible for an increasing proportion of community-acquired infections across Canada. All physicians are familiar with the present day reality of community-associated methicillinresistant Staphylococcus aureus (MRSA) in skin and soft tissue infections as well as the precipitous rise of resistance to fluoroquinolones and co-trimoxazole in community uropathogens such as $E$. coli.

"Do Bugs Need Drugs?" is a community education program designed to address antibiotic resistance by decreasing the inappropriate use of antibiotics. Beginning as a small six-month pilot in 1997 in Grande Prairie, Alberta, the program expanded to Edmonton in 2000 and to all of Alberta and to British Columbia in 2005. Resources have been developed for physicians, pharmacists, nurses and the public, including children, their parents and caregivers, teachers, employers and workers, and long-term care facilities. Programs are supported by print materials, a website, television and transit advertising, and a twitter account. The Do Bugs Need Drugs website is bilingual and some print materials are available in multiple languages (1).

British Columbia has seen reductions in the rate of community prescribing at the population level, especially for respiratory tract infection in children, the major early target of the program (2). In Alberta, consistent reductions in antibiotic prescribing in long-term care centres have been achieved with implementation of an antimicrobial stewardship strategy that includes education for staff and feedback on antibiotic prescribing rates (unpublished data; Mary Carson). The objective of this article is to summarize the key strategies of this community-based antimicrobial stewardship program. 


\section{Four key strategies}

The success of Do Bugs Need Drugs can be attributed in part to four strategies: consistent messaging, networking, aligning interests, and containing costs.

\section{Consistent messaging}

Three key messages were established when the program was created and continue to be the focus of educational efforts today. These have been incorporated into program materials at all levels from health care professionals to preschoolers:

- Handwashing is the best way to stop the spread of infections.

- $\quad$ Bacteria and viruses are different and antibiotics do not work against viruses.

- Use antibiotics wisely to limit development of antibiotic resistance.

While it is relatively easy, for example, to instruct young children how to properly wash their hands, teaching antibiotic resistance is considerably more challenging. However, even young children can learn about the different kinds of germs and that antibiotics will "go into" bacteria but not viruses. We feel that consistency and repetition of these key messages has been central to successfully mounting a public education campaign.

\section{Networking}

Networking in an essential activity of Do Bugs Need Drugs as it is through community partners that the program was initiated and continues to be delivered. Much of this has been achieved through the Do Bugs Need Drugs committee structures in Alberta and British Columbia. Initially these committees included a medical microbiologist and infectious disease specialist, an antimicrobial utilization and infectious diseases pharmacist, a pharmacist from the pharmaceutical industry, a nurse associated with the clinical practice guideline program, a clinical professor from a faculty of pharmacy, and a representative from a health NGO. Subsequently, epidemiologists, members of professional colleges, and health practitioners involved with public health, long-term care and occupational health have become part of the team.

Committee members have been exceptionally dedicated to developing the Do Bugs Need Drugs program and have given their time without expectation of remuneration or personal recognition. They have also engaged a wide network of community partners, including government ministries, health care professional organizations, academic institutions, industry and businesses, long-term care facilities, schools, teachers, child care groups, church groups, youth organizations and individual citizens, all of which have assisted with program dissemination.

\section{Aligning interests}

Because Do Bugs Need Drugs relies to a large extent on partnerships with external organizations/groups for program delivery, it is important to identify partners with aligned interests and then to develop or tailor programs to meet their needs.

One example is the Grade 2 Program, which is designed to be used by guest presenters in the classroom. Training for the presenters, a teaching kit and resources for the Grade 2 students and school are provided by Do Bugs Need Drugs, while the program is delivered primarily by nursing and medical students as part of their community health requirement. This model has the advantage of educating both young children and future health care workers and has been a core activity of Do Bugs Need Drugs since 1997.

The Grade 2 Program depends on maintaining collaborative relationships with nursing and medical faculties and ensuring that Do Bugs Need Drugs materials are aligned with the academic expectations of the college or 
university. This approach has been used with the majority of our programs as it facilitates dissemination of key messages while providing a service that addresses the needs of partner organizations.

\section{Containing costs}

The cost of delivering Do Bugs Need Drugs provincially in Alberta is about five cents per Albertan per year. Several factors contribute to cost containment, including reliance on partner organizations for program delivery and economies of scale associated with implementation of Do Bugs Need Drugs programs in both Alberta and British Columbia. An example of reliance on partner organizations is-instead of direct mailing to businesses in Alberta about the availability of materials for the workplace, we have partnered with the Alberta Health Services Workplace Immunization Program and Victorian Order of Nurses to distribute packages of employer/worker materials to businesses in conjunction with workplace influenza clinics. With respect to economies of scale, Do Bugs Need Drugs programs in Alberta and British Columbia enjoy reduced printing and administrative costs as a result of combined print orders and use of the same teaching kits and web resources in both provinces.

\section{Discussion}

Program development and delivery is not easy. Antibiotic resistance is a challenging concept not only for public education but for health care professionals as well. Overcoming the view that antibiotic resistance is not an urgent issue can be daunting and requires perseverance and endurance. We have found, however, that over time the public has become more aware of what antibiotic resistance is and how it is caused. In $1998,27 \%$ of respondents to a public survey were able to correctly define antibiotic resistance. In a 2014 public survey in $\mathrm{BC}, 77 \%$ of those surveyed were able to attribute antibiotic resistance to misuse or overuse. The growing body of evidence about the global and personal risks associated with antibiotic use and the increased willingness of the scientific and medical community to discuss these risks in the media are important factors in heightening public awareness.

Following excellent international work in the area of antimicrobial resistance $(3,4)$, the Public Health Agency of Canada has led the development of a Federal Action Plan on Antimicrobial Resistance and Use (5). Importantly, the Canadian strategy addresses antibiotic use in agriculture and the need to develop an agri-food, antimicrobial usage tracking system (5). We are encouraged by this recent activity and are hopeful that the momentum behind antimicrobial stewardship campaigns in Canada continues to grow.

\section{Conclusion}

Antimicrobial stewardship can be supported at the local level through public education campaigns such as Do Bugs Need Drugs. The opportunities for initiating antimicrobial stewardship campaigns within a community are innumerable and there is no absolute right or wrong approach. What is most important is to get started.

\section{Acknowledgements}

The authors wish to acknowledge Edith Blondel-Hill and Susan Fryters for their seminal work in establishing the "Do Bugs Need Drugs?" program.

\section{Conflict of interest}

None

\section{Funding}

Alberta Health Services for funding in Alberta; BC Ministry of Health, Pharmaceutical Services Branch, for funding in British Columbia. 


\section{References}

(1) Do Bugs Need Drugs? A Community Program for Wise Use of Antibiotics. http://www.dobugsneeddrugs.org/about/

(2) BC Centre for Disease Control. Antibiotic Utilization in the Province of British Columbia, 2013. Vancouver, BC: BC Centre for Disease Control; 2014 Nov. http://www.bccdc.ca/NR/rdonlyres/C3BFA092-7C55-46ED-BFC93CD91E593A5A/0/Reportonantibioticutilization2013_Nov12_FINAL.pdf

(3) World Health Organization (WHO). The evolving threat of antimicrobial resistance: Options for Action, 2012. Geneva: WHO; 2012. http://whqlibdoc.who.int/publications/2012/9789241503181_eng.pdf?ua=1

(4) Government of the United States. National Action Plan for Combating Antibiotic-resistant Bacteria. Washington: Government of the United States; 2015 Mar. https://www.whitehouse.gov/sites/default/files/docs/national_action_plan_for_combating_antiboticresistant_bacteria.pdf

(5) Government of Canada. Federal Action Plan on Antimicrobial Resistance and Use in Canada: Building on the Federal Framework for Action. 2015 Mar 31.

http://healthycanadians.gc.ca/publications/drugs-products-medicaments-produits/antibiotic-resistanceantibiotique/action-plan-daction-eng.php. 